\title{
Vaccination and Therapeutics: Responding to the Changing Epidemiology of Yellow Fever
}

Amanda Makha Bifani, MSc ${ }^{1}$

Eugenia Z. Ong, $P h D^{1,2}$

Ruklanthi de Alwis, MPH, PhD ${ }^{1,2, *}{ }^{*}$

\author{
Address \\ ${ }^{*}, 1$ Program in Emerging Infectious Diseases, Duke-NUS Medical School, Singapore, \\ Singapore \\ Email: rukie.dealwis@duke-nus.edu.sg \\ ${ }^{2}$ Viral Research and Experimental Medicine Centre @ SingHealth Duke-NUS (VI- \\ REMiCS), Singapore, Singapore
}

Published online: 6 November 2020

(C) Springer Science+Business Media, LLC, part of Springer Nature 2020

This article is part of the Topical Collections on Viral Infections

Keywords Yellow fever · Epidemiology · Vaccination - Therapeutics · Immunity · Outbreak control

\section{Abstract}

At the turn of the nineteenth century, yellow fever (YF) was considered the most dangerous infectious disease with high case fatality. Subsequent, mass vaccination campaigns coupled with widespread elimination of the YF mosquito vector significantly decreased YF cases and reduced outbreaks to the tropical and subtropical forested regions of Africa and South America. However, recent (2016) large outbreaks in Angola, Democratic Republic of Congo (DRC), and South-Eastern Brazil, where previously had been demarcated as low-risk regions, have highlighted the possibility of a rapidly changing epidemiology and the potential re-emergence of yellow fever virus (YFV). Furthermore, the first-ever importation of YFV into Asia has highlighted the potential fear of YFV emerging as a global threat. In this review, we describe the changing epidemiology of YF outbreaks, and highlight the use of public health policies, therapeutics, and vaccination as tools to help eliminate future YFV outbreaks.

\section{Introduction}

Yellow fever (YF) is a re-emerging acute viral disease that less than a century ago was considered the most dangerous infectious disease in the world [1]. YF is caused by the etiological agent, yellow fever virus (YFV), which belongs to the flavivirus genus of arboviruses. YFV exists in circulation between humans, non-human primates 
(NHPs), and several species of mosquito vectors [2]. The presence of epizootic transmission means that YFV cannot be eradicated from the world. However, preventative vaccination campaigns with live-attenuated YFV vaccines along with widespread mosquito elimination programs have proven to be efficient at substantially reducing YF cases and outbreaks [3]. Individuals infected with YFV can present with a wide spectrum of symptoms, ranging from asymptomatic infections to death, with severe cases displaying high fever, dysfunction of multiple organs (including the liver, kidneys, and heart), or hemorrhage [4]. The ratio of subclinical (i.e., asymptomatic to mild symptoms) to severe cases is estimated to be $1-70$, indicating that severe cases represent only "the tip of the iceberg" [5]. Mathematical models have also predicted over 200,000 annual cases of YF in high risk areas, with case fatalities ranging from 20 to $80 \%[1,6,7]$. However, due to the sporadic nature of YF outbreaks, the lack of rapid diagnostics, and the high serological crossreactivity among co-circulating flaviviruses has meant that confirmed diagnosis is limited to reference laboratories where viral RNA can be detected using reverse transcription (RT)-PCR [8•, 9]. Therefore, quantifying the true global burden of YF disease and infections has been challenging.

The complex transmission pattern of YFV between humans, non-human primates, and its mosquito vector has shaped the epidemiology of YF. Genotypes and transmission patterns of YFV are highly divergent between Africa and South America [2]. In South America, YFV strains circulate in two different cycles (Fig. 1a), i.e., (1) a sylvatic cycle between non-human primates (NHP) and rainforest-dwelling mosquitoes of the Haemagogus and Aedes species, and (2) an urban cycle where in rare opportunistic forest mosquito-feeding events YFV infect humans and spreads through urban human populations via the urbanized mosquito, Aedes aegypti. Africa observes an additional YFV transmission cycles known as the intermediate cycle (Fig. 1b), where anthropophilic mosquitoes in the savannah regions feed on both NHPs and humans leading to constant transmission of YFV between mosquito, NHPs, and humans [10]. Interestingly, compared to NHP species from Africa, NHP species in South America are far more susceptible to YFV infection with high mortality $[11,12]$.

YFV exists as seven genotypes (or major lineages), with 5 circulating in Africa and 2 in South America. Phylogenetic studies show that the African lineages have a common ancestor that then spread to discrete geographical locations and further evolved to establish the five genotypes (1) West African I, (2) West African II, (3) East and Central African, (4) East African, and (5) Angola $[1,13]$. The South American genotypes I and II are thought to have evolved from the West African strains during the time of the slave trade when YFV spreads to the Americas [14]. Unlike flaviviruses such as dengue virus (DENV), YFV is highly stable with slow epidemic and evolutionary rates $[15,16]$. Due to slow evolutionary rates and constant spillover, the phylogeny of YFV isolates from NHP are genetically similar to isolates from human outbreaks $[12,17,18]$. Consequently, in South America, regular surveillance of YFV outbreaks in NHP populations can be used as an epidemic preparedness strategy $[12,19]$. In addition to active surveillance, elimination of future YF outbreaks requires a comprehensive understanding of the current epidemiology and the influence of control and prevention methods, such as vaccination.

\section{Current Epidemiology: Appearance of YF in Low or No} Endemic Regions

In the eighteenth and 1nineteenth centuries, YFV led to high rates of mortality in Africa and the Americas $[3,20]$. Fortunately, in the mid-1960s, Max Theiler and colleagues created the earliest versions of the liveattenuated yellow fever vaccine $[3,21]$. Subsequently, widespread mass vaccination campaigns and mosquito control efforts significantly reduced YF outbreaks. Past public health successes led to a lax in maintaining local YF vaccination coverage leading to waning herd immunity and an eventual re-emergence of large outbreaks in

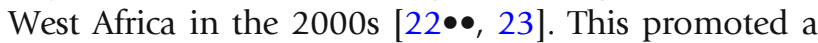
collaborative effort by the World health Organization (WHO), the United Nations Children's Fund (UNICEF), Global Alliance for Vaccines and Immunization (GAVI), and 47 YFV endemic countries to create the Yellow Fever Initiative (YFI) in 2006 [24]; an initiative that was primarily focused on mass YF vaccination campaigns and introduction of the vaccine into childhood immunization programs in high risk regions. In an emergency preparedness effort, the new initiative also set up a global stockpile of YF vaccines [25]. While implementation of YFI eliminated YF outbreaks, this was short-lived as the emergence of major YF outbreaks in 2016 in regions not previously characterized as high-risk for YF casted doubts on the YFI strategy [7].

In Africa prior to 2016, YFV outbreaks occurred predominantly in West and East Africa, with low or no endemicity in Central Africa (Fig. 1b). In 2013, highly 
a

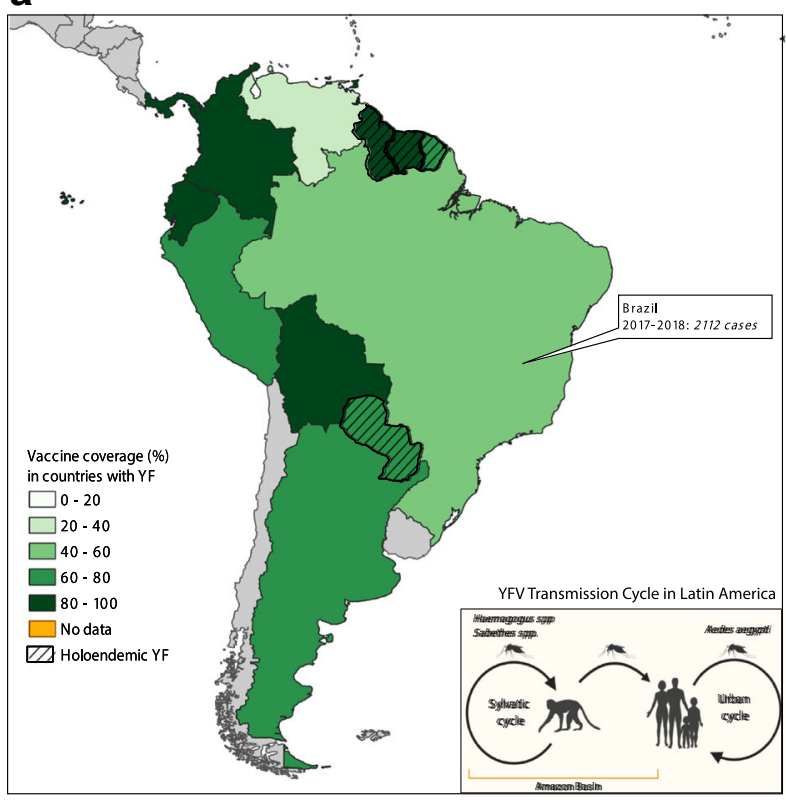

b

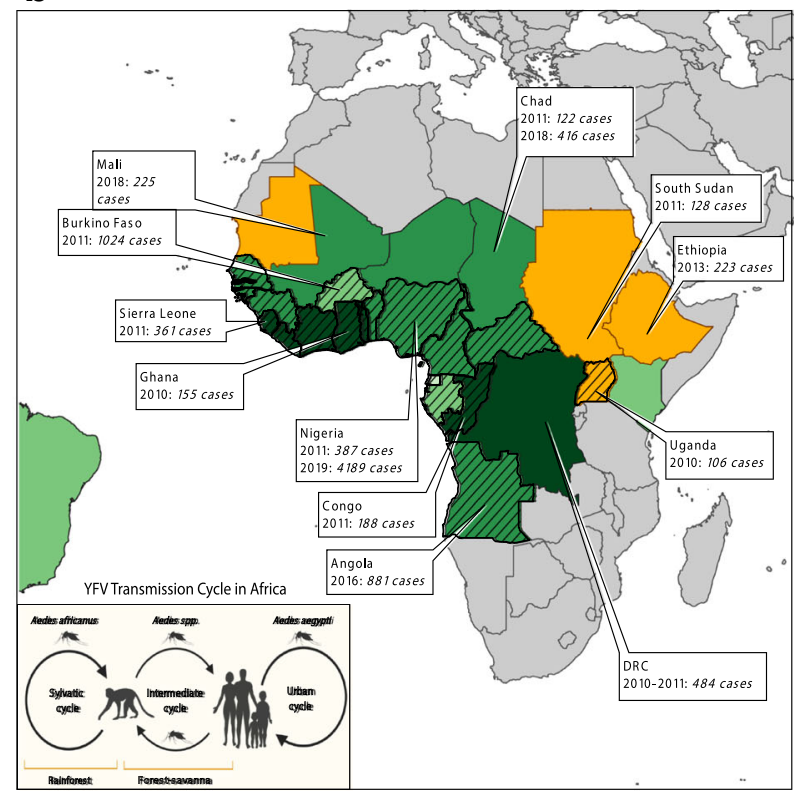

Fig. 1. Map displaying major outbreaks in the past decade and the vaccine coverage in countries reported as "high risk" for YFV by WHO. Major outbreaks are defined as greater than 100 cases of YF reported to WHO. All data for global YF cases and vaccine coverage were accessed from most current estimates on the WHO website (https://www.who.int/news-room/fact-sheets/detail/yellowfever)

endemic regions in Africa were estimated to have a YF burden of 130,000 cases per year, which accounted for greater than $90 \%$ of the global burden [7]. It had also been observed that ambient or environmental temperatures and rainfall patterns could sustain mosquito vectors and promote the spread of YFV to regions of low endemicity [26]. In December 2016, YF cases were detected in Luanda, the capital city of Angola, which was previously categorized as a low-risk region for YF. The epidemic rapidly spread from Luanda to other urban populations in Angola and crossed into the neighboring country, Democratic republic of Congo (DRC) [27]. Modeling studies predict that YFV transmission was continued for longer than usual due to the rain patterns affected by the 2016 EL Niño phenomenon [28]. Emergency vaccines were deployed to affected regions, but by the mid-2016, the entire global stockpile of YF vaccine doses was exhausted, and fractional dosing of the YF vaccine was implemented as an emergency measure [29]. By November 2016, the WHO had confirmed 962 cases and 393 deaths related to this YFV outbreak. Since Angola and DRC were previously classified as lowrisk for YFV transmission, these countries had not qualified for the previous vaccination campaigns within the YFI [24]. Low herd immunity, due to low YF vaccination coverage, facilitated the large 2016 outbreak in Angola and DRC. As a response, the WHO revised its YFI program and implemented the new "EYE" strategy, with the goal of eliminating all global YF epidemics by 2026 [30••]. Since 2016, there have been outbreaks of 100 or more cases reported to WHO from Mali and Chad (2018) and Nigeria (2019). As a step towards eliminating future YF outbreaks, mass vaccination campaigns are currently being carried out in YF endemic regions where

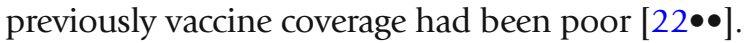

In South America, case fatality rates from YFV infections are more than double that in Africa (40$60 \%$ versus $20 \%$ ) [1]. Therefore, under the YFI program, preventative vaccination campaigns in high risk YF regions significantly reduced YFV outbreaks in humans until no major outbreak (i.e., greater than 100 cases) was detected between 2010 and 2015 in Latin America. Nevertheless, in 2016, a large YF outbreak occurred in regions of Brazil that had previously had low or no risk of YF infections and therefore, low vaccination coverage [17]. Phylogenetic tracking of the epidemic strains identified the originating dissemination event to have occurred in "Central-West" region in the Brazilian state of Goiás about 2 years prior to the 
outbreak [31, 32]. The epidemic then moved into the state of Minas Gerais, from where it moved along two different routes (route 1 towards the east and route 2 towards the south-east) into peri-urban regions of the Brazilian megapolis, São Paulo and Rio de Janeiro [31]. This led to an exponential increase in transmission leading to over 2000 confirmed cases and over 600 deaths. Molecular analysis confirmed that the 2016-2017 epidemic strain was within the South American (SA) 1 genotype and that the epidemic resulted from direct sylvatic spillover to humans with the absence of sustained YFV transmission within the urban cycle [19, 32, 33]. Some studies have attributed the 2016 YF epidemic in YF non-endemic regions of Brazil to increased virulence of the new virus strain [31, 32, 34]; others have blamed changing aspects of population and ecology, such as the rise in population density [34], the geographical expansion of the peri-urban mosquito Aedes albopictus [35], human encroachment into NHP habitats [36], and global warming [37]. Since the factors that promote emergence of YFV in low or non-endemic regions are not well understood, currently, we are unable to predict the next YFV outbreak in these regions.

It is unclear why Asia has never reported endemic YFV transmission, despite similar flaviviruses (such as DENV and zika virus) circulating in this region. However, Asia is the home to over 4 billion individuals, contains widespread prevalence of the YF mosquito vector (Aedes aegypti), and has no heard immunity against YFV (due to lack of natural circulation and vaccination) [38]. Therefore, increased movement of individuals between Asia and Africa and/or South America pose a real fear that YFV might spread to Asia [39]. In 2016, eleven Chinese working in Angola returned to China and imported YFV for the first time into Asia [40, 41]. Despite mandatory YF immunization requirements at least 10 days prior to travel to YFV-endemic regions outlined by the International health regulations (IHR), 5/11 of the Chinese workers had no YF vaccination record, while $4 / 11$ had been vaccinated $<14$ days of acquiring symptoms $[42,43]$. As links between Asia and Africa and/or South America increase, the threat of YFV spreading to Asia becomes real. Therefore, there is an urgent need to invest in research for new YFV rapid diagnostics, therapeutics, and vaccines. Furthermore, as part of YFV epidemic preparedness, it is essential that Asian countries also strengthen YFV surveillance, implement mosquito elimination programs, and put in place measures to rapidly manufacture and deploy vaccines.

\section{YF Vaccine: Our Imperfect but the Strongest Control Strategy}

In the live-attenuated YF vaccine, 17D remains the single best control measure against YFV infection and is widely regarded as one of the most successful vaccines ever developed. The 17D strain was developed in 1936 through the tedious process of serial passaging in chicken tissue of the wildtype YFV Asibi strain isolated from a patient in Ghana. There are currently three sub-strains (17D-204, 17D-213, and 17DD) licensed for use in individuals aged 9 months to 60 years old, residing in or traveling to YF-endemic regions. For all substrains of YF vaccines, antibodies against YFV are currently the gold standard when examining correlates of protection against future infection [44, 45]. Protective titers were originally determined by passive immune transfer and subsequent challenge with YFV in monkeys [46]. A log neutralization index (LNI) of 0.7 or higher is protective. However, calculating LNI requires pre- and post-vaccine serum, which is not always feasible in the context of natural infection or mass vaccination. Consequently, plaque reduction neutralizing titer (PRNT) assays have replaced LNI measurements, where an 80 or $90 \%$ PRNT (i.e., $\mathrm{PRNT}_{80} \mathrm{PRNT}_{90}$ ) is used to indicate protection [47]. However, both YFV LNI and PRNT are slow, tedious, not standardizable, require experienced laboratory staff, and are limited to biosafety level-3 containment facilities [48]. Therefore, currently, we are urgently in need of new rapid, high-throughput, easy-to-use techniques that can be used to accurately assess protective antibodies following vaccination.

Recent epidemiological studies have expanded our understanding of factors that affect YF vaccinationinduced human antibody responses (Fig. 2). Historically, YF vaccine studies conducted in health adults demonstrated that a single subcutaneous dose elicits a remarkably high seroconversion (nearly 100\%) [49-51]. However, recent studies show that age has a large influence on seroconversion rates, as observed by the high rates of vaccine failure in infants (9-12 months) and the elderly $(>60$ years) $[52 \bullet \bullet, 53]$. In particular, seroconversion rates of children who received the YF vaccine in conjunction with the routine childhood vaccines for measles-mumps-rubella (MMR) had a lower seroconversion rates $(69 \%)$ compared to children who received the YF vaccine alone $(\sim 85 \%)[54,55]$. Additionally, seroconversion rates in non-endemic countries 


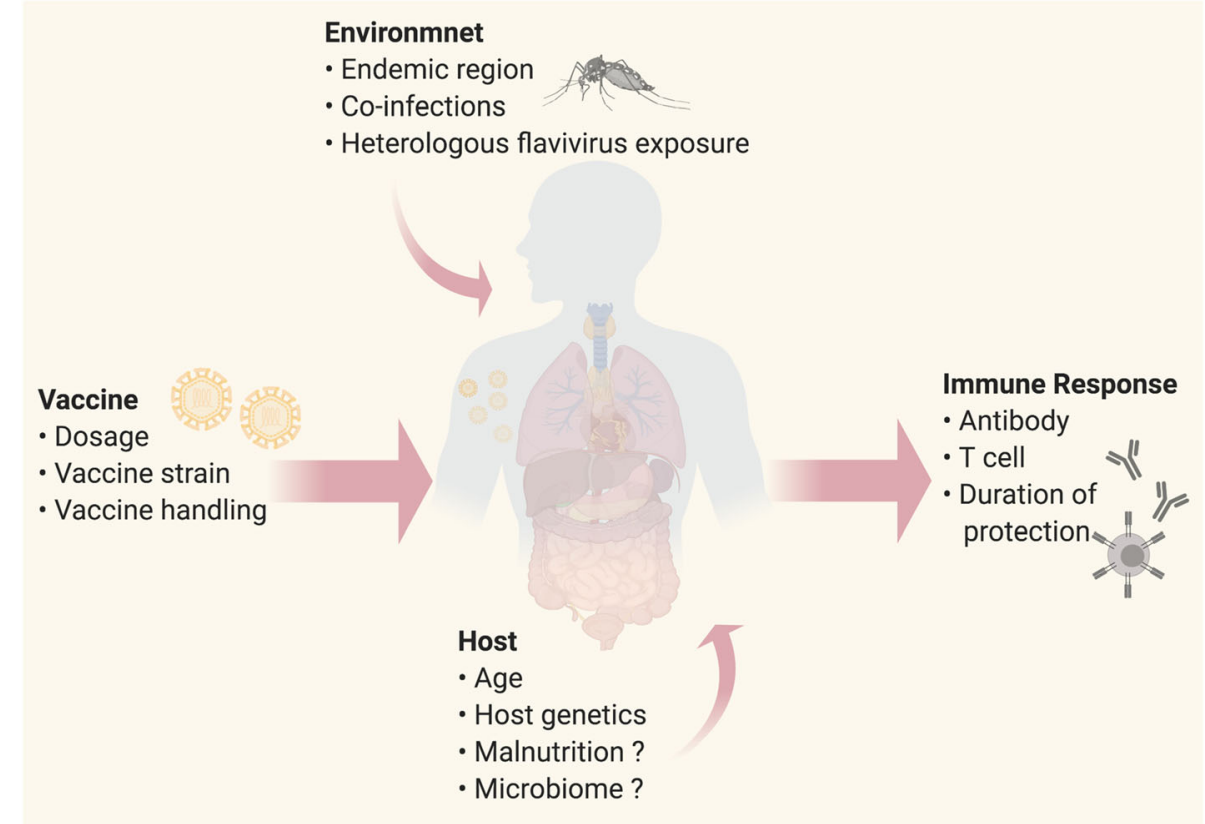

Fig. 2. Determinants of yellow fever live-attenuated vaccine immunity. Schematic diagram illustrating different elements of the YF vaccine (dosage, vaccine strain, vaccine handling, i.e., cold chain), environment (endemic vs non-endemic regions, co-infection, heterologous flavivirus exposure), and host (age, genetics, microbiome) that could influence host immune response outcome to YF vaccination. Created with BioRender.com

(98.9\%) tend to exceed seroconversion rates in endemic countries $(94.2 \%)$ [50]. Whether the observed difference in seroconversion rates between endemic versus nonendemic countries is due to host genetics [56], exposure to other circulating flaviviruses [1], microbiome, and additional co-infections that alter host immune response $[57,58]$ or host nutritional status remains to be elucidated.

Duration of immunity and the need for a booster has been highly debated in past years, with arguments for [59] and against a YF booster vaccination [60]. In controversial studies carried out in non-endemic countries, it had been reported that $17 \mathrm{D}$ offer long-term sero-protection lasting up to 30-40 years post vaccination [61, 62]. Therefore, in 2012, the WHO Strategic Advisory Group of Experts on immunization declared that a single dose of YF vaccine was sufficient for long-term protection in 2012 [63]. However, more recently, a substantial number of studies have highlighted that neutralizing antibody titers and cellular immunity wane with time [59, 64]. In adults, sero-positivity decreases at just 59 years post vaccination from 100 down to $82 \%$ [59]. This trend of waning immunity is further exaggerated at around 10-year post-vaccination with sero-protection dropping under $80 \%$ in both endemic $[59,65]$ and non-endemic cohorts [64]. Furthermore, waning immunity in children vaccinated between 9 to 12 months of age is more severe with only $\sim 40 \%$ retaining long-term sero-protection ( $\sim 6$ years post vaccination) in Brazil

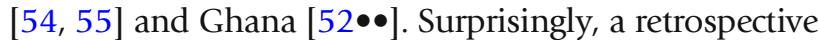
study in Brazil between 1973 and 2008 noted that the number of hospitalized YF cases with and without records of past YF vaccination (52\% vs $45 \%$, respectively) was not significantly different [47]. In fact, vaccination coverage of up to $95 \%$ maybe required for high risk regions to eliminate yellow fever outbreaks [66]. These disturbing observations are further evidence of waning YF vaccine immunity over time, and thus provide strong support that a YF booster vaccination is needed (i.e., a 10-year vaccination booster for travelers [67] and a 45 year booster for children vaccinated during their first year of life).

Following the global shortage of YF vaccine supplies in 2016, in an effort to maximize vaccination coverage and to curtail the epidemics in Angola and DRC, public health officials proceeded to conduct fractional dosing of the remaining YF vaccines [68]. Studies in Rhesus macaques showed fractional dosing of $17 \mathrm{D}$ to be effective 
in protecting against wildtype Asibi in a challenge model [46]. In humans, a 10-fold lower dose of YF17D provoked a similar cytokine profile, viremia, and PRNT response as a standard dose [69]. Compared to full dose, fractional dosing of YF17D stimulated similar seroconversion rates [70] that persisted for 10 months to 1 year post-vaccination $[71,72]$. Long-term protection was observed in $98 \%$ of patients receiving a fractional dose in a non-endemic setting [73], while only $85 \%$ of recipients in an endemic region maintained lasting immunity [74]. Overall, the decision to use a fractional dosing in an outbreak setting was supported by the prediction that during the 2016 YF epidemic in Angola, a lack of fractional dosing would have led to a 5.1-fold increase in mortality due to YF [28]. However, our understanding of the effect of fractional dosing on waning immunity, on vaccine shelf-life, on the antibody responses in different age groups and genetic backgrounds, is limited [68]. Hence, it is imperative that we continue to improve our evidence base to understand the effect of YF vaccine dose-fractionation on protection against YFV infections.

The role of $\mathrm{T}$ cell immunity in yellow fever vaccination remains unclear. Not surprisingly, YF vaccination induces strong $\mathrm{CD} 4^{+}$and $\mathrm{CD} 8^{+} \mathrm{T}$ cell responses, which persists for several years following vaccination $[55,75]$. CD $4^{+} \mathrm{T}$ cell expansion occurs earlier and with a greater magnitude after vaccination than $\mathrm{CD}^{+} \mathrm{T}$ cells $[62,75-77]$. Epidemiological evidence show that seroconversion rates drop and immunity wanes rapidly following YF vaccination of HIV-positive patients, whose $\mathrm{CD} 4^{+} \mathrm{T}$ cell response is impaired [78]. While these studies suggest that $\mathrm{T}$ cells contribute to maintenance of seroprotection and flaviviral clearance, it is likely that $\mathrm{T}$ cell immunity alone is insufficient to confer protection [47]. Furthermore, immunization with the chimeric flavivirus vaccine on a YF17D backbone of non-structural proteins did not offer protection when challenged with YFV [79]. On the other hand, passive transfer of antibodies was sufficient for protection against wildtype infection [44, 46].

Therefore, it is likely that although both B and T cells play a role in establishing viral clearance, antibody responses play a more dominant role in eliciting YF vaccine-induced protection.

In terms of vaccine safety, YF vaccination has been associated with several rare but related adverse events (AEs) particularly in the elderly ( $\geq$ 60 years old) [80-82]. Vaccine-associated neurotropic disease (YFV-AND) causes neurological complications that are typically not fatal. However, vaccine-associated viscerotropic disease (YFV-AVD) results in infection that resembles natural YF disease accompanied by a high fatality rate. Virus isolated from YFV-AVD patients does not have any mutations in the $17 \mathrm{D}$ genome $[83,84]$; thus, the driver of YFV-AVD remains unknown. AEs tend to be reported more frequently in non-endemic countries, possibly as result of under-reporting in endemic countries [47]. Additionally, host genetics [56], egg allergies (due to the manufacturing process) [80], and an active immune environment [58] have been linked with disease outcome and could contribute to frequency of adverse events experience by different populations. At the same time, YF vaccines unknowingly administered to pregnant women were immunogenic and elicited varying degrees of sero-protection without harming or transplacentally infecting the fetus, illustrating the safety profile of YF vaccines during pregnancy $[85,86]$. Therefore, given the low incidence of AEs in high risk areas, and the large risk of mortality associated with YF infections, the threat from wildtype YFV infections outweighs that from YF vaccineassociated AEs [80].

The EYE's initiative aims to eliminate YF through vaccination of 1 billion people living in at-risk areas by 2026. Unfortunately, these efforts have been thwarted by vaccine shortages. The ambitious EYEs initiative assumes that a single dose confers life-long immunity, yet as discussed above, this is likely not the case. It is estimated that 90 million doses of the $17 \mathrm{D}$ vaccine are needed every year. Due to the slow, tedious, and low-yielding process of manufacturing YF vaccine doses in chicken embryos [1], the production capability is limited and unable to meet increased demands during an outbreak or booster vaccination campaigns. Efforts are being made to develop new vaccines with higher yields and increased safety profiles [87-90]. The most promising is a cell culture (Vero cell)based production of an inactivated YFV vaccine [91]. The inactivated vaccine showed promising results in clinical trials [91], but was less immunogenic than the live-attenuated YF vaccines and required a two-dose schedule with an adjuvant. Ideally, a new cell culture method for YF17D that 
increases yields and cuts production time, while maintaining efficacy, genomic stability, and safety, would be required to meet the world's growing demands for the vaccine.

\section{Possible Role for Therapeutics in YF Control?}

There are no approved therapeutics against YFV infections and treatment of YF disease is reliant on supportive care to alleviate disease symptoms. Although its initial clinical presentation may be benign, $15 \%$ of symptomatic patients can rapidly progress to severe yellow fever that requires intensive care support [92]. This includes the prophylactic use of anti-convulsant drugs to reduce the frequency of seizures, and use of intravenous proton pump inhibitors to treat gastric bleeding [93]. While there are licensed YF vaccines, as discussed above, mass immunization programs have been hampered by global vaccine shortages. Against this backdrop, an effective antiviral against YFV could be used as a treatment (to significantly improve the clinical outlook for patients with severe YF) and also as an outbreak control strategy (i.e., as a prophylaxis when YF vaccine is in short supply).

Drug repurposing has been adopted as a strategy for identifying de-risked compounds that have demonstrated safety profile in preclinical models or humans, with potential time saved for drug development [94]. Although there are currently no approved antivirals for the treatment or prevention of $\mathrm{YF}$, there are several repurposed candidates in the drug development pipeline. Galidesivir and
Sofosbuvir are both nucleoside analogue antivirals originally used to treat chronic hepatitis C infections, which have emerged as promising antivirals for the treatment of YF [95, 96]. Both Galidesivir and Sofosbuvir were found to inhibit YF replication in vitro and in vivo in rodent animal models $[97,98]$. Interestingly, therapeutic administration of Galidesivir allowed development of neutralizing antibodies against YFV that completely protected against secondary YFV challenge, highlighting that the use of these drugs will not hamper development of adaptive immunity [97]. Following completion of Phase IA clinical trial to evaluate safety and tolerability of intravenous Galidesivir in healthy volunteers, a Phase IB study that aims to evaluate antiviral effects of intravenous Galidesivir in YF patients is currently ongoing (clinicaltrials. gov NCT03891420). Recently, two YFV-infected patients presenting with acute liver failure were treated off-label with Sofosbuvir for a week as compassionate use and displayed marked reduction in viremia and improved liver function [99]. Furthermore, in recent years, virus-neutralizing monoclonal antibodies (mAbs) have also been recognized as a treatment modality for YF [100]. The candidate that is most advanced in clinical development is TY014, which has currently completed Phase 1A/ 1B safety trials (clinicaltrials.gov NCT03776786). As antivirals against YFV become licensed for use in patients and travelers, it is hoped that these will contribute to both treatment of YF disease and the control of YF outbreaks.

\section{Concluding Remarks}

The re-emergence of outbreaks in low-risk areas and importation of cases to Asia indicate the rapidly changing epidemiology of YF. As part of epidemic preparedness, efforts should be directed at enhancing YF surveillance, diagnostic capabilities of laboratories and improving vaccine coverage, even in low endemic regions. While the live-attenuated YF vaccine is highly efficacious, vaccine supply shortfalls due to limited production suggest that the development of new vaccines and improved manufacturing technologies will be urgently needed to meet increased worldwide demand. Lastly, a two-pronged approach that combines vaccination and therapeutic interventions will be paramount to prevent the re-emergence of future YF outbreaks. 


\section{References and Recommended Reading}

Papers of particular interest, published recently, have been highlighted as:

- Of importance

$\bullet \quad$ Of major importance

1. Monath TP, Vasconcelos PF. Yellow fever. J Clin Virol. 2015;64:160-73. https://doi.org/10.1016/j.jcv.2014.08. 030.

2. Staples JE, Monath TP. Yellow fever: 100 years of discovery. JAMA. 2008;300(8):960-2. https://doi.org/10. 1001/jama.300.8.960.

3. Frierson JG. The yellow fever vaccine: a history. Yale J Biol Med. 2010;83(2):77-85.

4. Quaresma JA, Pagliari C, Medeiros DB, Duarte MI, Vasconcelos PF. Immunity and immune response, pathology and pathologic changes: progress and challenges in the immunopathology of yellow fever. Rev Med Virol. 2013;23(5):305-18. https://doi.org/10. 1002/rmv.1752.

5. Johansson MA, Vasconcelos PF, Staples JE. The whole iceberg: estimating the incidence of yellow fever virus infection from the number of severe cases. Trans R Soc Trop Med Hyg. 2014;108(8):482-7. https://doi.org/ $10.1093 /$ trstmh/tru092.

6. Shearer FM, Longbottom J, Browne AJ, Pigott DM, Brady OJ, Kraemer MUG, et al. Existing and potential infection risk zones of yellow fever worldwide: a modelling analysis. Lancet Glob Health. 2018;6(3):e270-e8. https://doi.org/10.1016/S2214109X(18)30024-X.

7. Garske T, Van Kerkhove MD, Yactayo S, Ronveaux O, Lewis RF, Staples JE, et al. Yellow fever in Africa: estimating the burden of disease and impact of mass vaccination from outbreak and serological data. PLoS Med. 2014;11(5):e1001638. https://doi.org/10.1371/ journal.pmed.1001638.

8. Domingo C, Charrel RN, Schmidt-Chanasit J, Zeller H, Reusken C. Yellow fever in the diagnostics laboratory. Emerg Microbes Infect. 2018;7(1):129. https://doi.org/ 10.1038/s41426-018-0128-8

The most recent review of the currently available methods to diagnose Yellow fever.

9. Ingelbeen B, Weregemere NA, Noel H, Tshapenda GP, Mossoko M, Nsio J, et al. Urban yellow fever outbreakDemocratic Republic of the Congo, 2016: towards more rapid case detection. PLoS Negl Trop Dis. 2018;12(12):e0007029. https://doi.org/10.1371/ journal.pntd.0007029.

10. Carrington CV, Auguste AJ. Evolutionary and ecological factors underlying the tempo and distribution of yellow fever virus activity. Infect Genet Evol. 2013;13:198-210. https://doi.org/10.1016/j.meegid. 2012.08.015.

11. Fernandes N, Cunha MS, Guerra JM, Ressio RA, Cirqueira CDS, Iglezias SD, et al. Outbreak of yellow fever among nonhuman Primates, Espirito Santo,
Brazil, 2017. Emerg Infect Dis. 2017;23(12):2038-41. https://doi.org/10.3201/eid2312.170685.

12. Silva NIO, Sacchetto L, de Rezende IM, Trindade GS, LaBeaud AD, de Thoisy B, et al. Recent sylvatic yellow fever virus transmission in Brazil: the news from an old disease. Virol J. 2020;17(1):9. https://doi.org/10.1186/ s12985-019-1277-7.

13. Mutebi JP, Wang H, Li L, Bryant JE, Barrett AD. Phylogenetic and evolutionary relationships among yellow fever virus isolates in Africa. J Virol. 2001;75(15):6999-7008. https://doi.org/10.1128/JVI. 75.15.6999-7008.2001.

14. Bryant JE, Holmes EC, Barrett AD. Out of Africa: a molecular perspective on the introduction of yellow fever virus into the Americas. PLoS Pathog. 2007;3(5):e75. https://doi.org/10.1371/journal.ppat. 0030075.

15. Chen C, Jiang D, Ni M, Li J, Chen Z, Liu J, et al. Phylogenomic analysis unravels evolution of yellow fever virus within hosts. PLoS Negl Trop Dis. 2018;12(9):e0006738. https://doi.org/10.1371/ journal.pntd.0006738.

16. Kum DB, Mishra N, Vrancken B, Thibaut HJ, WilderSmith A, Lemey P, et al. Limited evolution of the yellow fever virus $17 \mathrm{~d}$ in a mouse infection model. Emerg Microbes Infect. 2019;8(1):1734-46. https:// doi.org/10.1080/22221751.2019.1694394.

17. Figueiredo PO, Silva ATS, Oliveira JS, Marinho PE, Rocha FT, Domingos GP, et al. Detection and molecular characterization of yellow fever virus, 2017. Brazil Ecohealth. 2018;15(4):864-70. https://doi.org/10. 1007/s10393-018-1364-z.

18. Rezende IM, Sacchetto L, Munhoz de Mello E, Alves PA, FCM I, Adelino TER, et al. Persistence of Yellow fever virus outside the Amazon Basin, causing epidemics in Southeast Brazil, from 2016 to 2018. PLoS Negl Trop Dis. 2018;12(6):e0006538. https://doi.org/ 10.1371/journal.pntd.0006538.

19. Mares-Guia M, Horta MA, Romano A, Rodrigues CDS, Mendonca MCL, Dos Santos CC, et al. Yellow fever epizootics in non-human primates, Southeast and Northeast Brazil (2017 and 2018). Parasit Vectors. 2020;13(1):90. https://doi.org/10.1186/s13071-0203966-x.

20. Douam F, Ploss A. Yellow fever virus: knowledge gaps impeding the fight against an old foe. Trends Microbiol. 2018;26(11):913-28. https://doi.org/10. 1016/j.tim.2018.05.012.

21. Norrby E. Yellow fever and Max Theiler: the only Nobel Prize for a virus vaccine. J Exp Med. 2007;204(12):2779-84. https://doi.org/10.1084/jem. 20072290. 
22.• Shearer FM, Moyes CL, Pigott DM, Brady OJ, Marinho F, Deshpande A, et al. Global yellow fever vaccination coverage from 1970 to 2016: an adjusted retrospective analysis. Lancet Infect Dis. 2017;17(11):1209-17. https://doi.org/10.1016/S1473-3099(17)30419-X

A modeling study that describes the global YF cases and vaccine coverage over four decades.

23. Rogers DJ, Wilson AJ, Hay SI, Graham AJ. The global distribution of yellow fever and dengue. Adv Parasitol. 2006;62:181-220. https://doi.org/10.1016/S0065308X(05)62006-4.

24. WHO. Yellow fever initiative providing an opportunity of a lifetime. Geneva: Organization WH, editor; 2010.

25. WHO. International Coordination Group on Vaccine Provision for Yellow Fever. Geneva: Organization WH, editor; 2019.

26. Hamlet A, Jean K, Perea W, Yactayo S, Biey J, Van Kerkhove $\mathrm{M}$, et al. The seasonal influence of climate and environment on yellow fever transmission across Africa. PLoS Negl Trop Dis. 2018;12(3):e0006284. https://doi.org/10.1371/journal.pntd.0006284.

27. Kraemer MUG, Faria NR, Reiner RC Jr, Golding N, Nikolay B, Stasse S, et al. Spread of yellow fever virus outbreak in Angola and the Democratic Republic of the Congo 2015-16: a modelling study. Lancet Infect Dis. 2017;17(3):330-8. https://doi.org/10.1016/S14733099(16)30513-8.

28. Zhao S, Stone L, Gao D, He D. Modelling the largescale yellow fever outbreak in Luanda, Angola, and the impact of vaccination. PLoS Negl Trop Dis. 2018;12(1):e0006158. https://doi.org/10.1371/ journal.pntd.0006158.

29. World Health O. WHO position on the use of fractional doses - June 2017, addendum to vaccines and vaccination against yellow fever WHO: Position paper June 2013. Vaccine. 2017;35(43):5751-2. https://doi. org/10.1016/j.vaccine.2017.06.087.

$30 . \bullet$ WHO. Eliminate Yellow fever Epidemics (EYE): a global strategy, 2017-2026. In: Organization WH, editor. Geneva; 2017. p. 193-204.

The official WHO document outlining the EYE strategy formed in 2016.

31. Delatorre E, de Abreu FVS, Ribeiro IP, Gomez MM, Dos Santos AAC, Ferreira-de-Brito A, et al. Distinct YFV lineages co-circulated in the Central-Western and Southeastern Brazilian regions from 2015 to 2018. Front Microbiol. 2019;10:1079. https://doi.org/10. 3389/fmicb.2019.01079.

32. Faria NR, Kraemer MUG, Hill SC, Goes de Jesus J, Aguiar RS, Iani FCM, et al. Genomic and epidemiological monitoring of yellow fever virus transmission potential. Science. 2018;361(6405):894-9. https://doi. org/10.1126/science.aat7115.

33. Cunha MDP, Duarte-Neto AN, Pour SZ, Ortiz-Baez AS, Cerny J, Pereira BBS, et al. Origin of the Sao Paulo Yellow Fever epidemic of 2017-2018 revealed through molecular epidemiological analysis of fatal cases. Sci Rep. 2019;9(1):20418. https://doi.org/10.1038/ s41598-019-56650-1.
34. Possas C, Lourenco-de-Oliveira R, Tauil PL, Pinheiro FP, Pissinatti A, Cunha RVD, et al. Yellow fever outbreak in Brazil: the puzzle of rapid viral spread and challenges for immunisation. Mem Inst Oswaldo Cruz. 2018;113(10):e180278. https://doi.org/10.1590/ 0074-02760180278.

35. Couto-Lima D, Madec Y, Bersot MI, Campos SS, Motta MA, Santos FBD, et al. Potential risk of re-emergence of urban transmission of Yellow Fever virus in Brazil facilitated by competent Aedes populations. Sci Rep. 2017;7(1):4848. https://doi.org/10.1038/s41598-01705186-3.

36. Nava A, Shimabukuro JS, Chmura AA, Luz SLB. The impact of global environmental changes on infectious disease emergence with a focus on risks for Brazil. ILAR J. 2017;58(3):393-400. https://doi.org/10.1093/ilar/ ilx034.

37. Balogun EO, Nok AJ, Kita K. Global warming and the possible globalization of vector-borne diseases: a call for increased awareness and action. Trop Med Health. 2016;44:38. https://doi.org/10.1186/s41182-016-0039-0.

38. Wilder-Smith A, Lee V, Gubler DJ. Yellow fever: is Asia prepared for an epidemic? Lancet Infect Dis. 2019;19(3):241-2. https://doi.org/10.1016/S14733099(19)30050-7.

39. Glaesser D, Kester J, Paulose H, Alizadeh A, Valentin B. Global travel patterns: an overview. J Travel Med. 2017;24(4). https://doi.org/10.1093/jtm/tax007.

40. Wang L, Zhou P, Fu X, Zheng Y, Huang S, Fang B, et al. Yellow fever virus: increasing imported cases in China. J Inf Secur. 2016;73(4):377-80. https://doi.org/10. 1016/j.jinf.2016.07.003.

41. Wasserman S, Tambyah PA, Lim PL. Yellow fever cases in Asia: primed for an epidemic. Int J Infect Dis. 2016;48:98-103. https://doi.org/10.1016/j.ijid.2016. 04.025 .

42. Song R, Guan S, Lee SS, Chen Z, Chen C, Han L, et al. Late or lack of vaccination linked to importation of yellow fever from Angola to China. Emerg Infect Dis. 2018;24(7). https://doi.org/10.3201/eid2407.171868.

43. Wilder-Smith A, Leong WY. Importation of yellow fever into China: assessing travel patterns. J Travel Med. 2017;24(4). https://doi.org/10.1093/jtm/tax008.

44. Julander JG, Trent DW, Monath TP. Immune correlates of protection against yellow fever determined by passive immunization and challenge in the hamster model. Vaccine. 2011;29(35):6008-16. https://doi. org/10.1016/j.vaccine.2011.06.034.

45. Plotkin SA. Correlates of protection induced by vaccination. Clin Vaccine Immunol. 2010;17(7):1055-65. https://doi.org/10.1128/CVI.00131-10.

46. Mason RA, Tauraso NM, Spertzel RO, Ginn RK. Yellow fever vaccine- direct challenge of monkeys given graded doses of 17D vaccine. Appl Microbiol. 1973;25(4):6.

47. Amanna IJ, Slifka MK. Questions regarding the safety and duration of immunity following live yellow fever vaccination. Expert Rev Vaccines. 2016;15(12):151933. https://doi.org/10.1080/14760584.2016. 1198259. 
48. Jonker EF, Visser LG, Roukens AH. Advances and controversies in yellow fever vaccination. Ther Adv Vaccines. 2013;1(4):144-52. https://doi.org/10.1177/ 2051013613498954.

49. Hepburn MJ, Kortepeter MG, Pittman PR, Boudreau EF, Mangiafico JA, Buck PA, et al. Neutralizing antibody response to booster vaccination with the $17 \mathrm{~d}$ yellow fever vaccine. Vaccine. 2006;24(15):2843-9. https://doi.org/10.1016/j.vaccine.2005.12.055.

50. Jean K, Donnelly CA, Ferguson NM, Garske T. A metaanalysis of serological response associated with yellow fever vaccination. Am J Trop Med Hyg. 2016;95(6):1435-9. https://doi.org/10.4269/ajtmh. 16-0401.

51. Monath TP, Nichols R, Archambault WT, Moore L, Marchesani R, Tian J, et al. Comparative safety and immunogenicity of two yellow fever 17D vaccines (ARILVAX and YF-VAX) in a PHASE III multicenter, double-blind clinical trial. Am J Trop Med Hyg. 2002;66(5):8.

52.• Domingo C, Fraissinet J, Ansah PO, Kelly C, Bhat N, Sow $\mathrm{SO}$, et al. Long-term immunity against yellow fever in children vaccinated during infancy: a longitudinal cohort study. The Lancet Infectious Diseases. 2019;19(12):1363-70. https://doi.org/10.1016/ s1473-3099(19)30323-8

Recent longitudinal study describing the issue of waning longterm immunity following YF vaccination in children.

53. Camacho LA, da Silva FM, da Luz Fernandes Leal M, de Aguiar SG, Do Nascimento JP, Iguchi T, et al. Immunogenicity of WHO-17D and Brazilian 17DD yellow fever vaccines- a randomized trial. Rev Saude Publica. 2004;38(5):8.

54. de Noronha TG, de Lourdes de Sousa MM, Geraldo Leite Ribeiro J, Campos Lemos JA, Maria Barbosa de Lima S, Martins-Filho OA, et al. Duration of postvaccination humoral immunity against yellow fever in children. Vaccine. 2019;37(48):7147-54. https://doi. org/10.1016/j.vaccine.2019.09.051.

55. Campi-Azevedo AC, Reis LR, Peruhype-Magalhaes V, Coelho-Dos-Reis JG, Antonelli LR, Fonseca CT, et al. Short-lived immunity after 17DD yellow fever single dose indicates that booster vaccination may be required to guarantee protective immunity in children. Front Immunol. 2019;10:2192. https://doi.org/10. 3389/fimmu.2019.02192.

56. Blake LE, Garcia-Blanco MA. Human genetic variation and yellow fever mortality during 19th century U.S. epidemics. MBio. 2014;5(3):10.1128/.

57. Teo TH, Lum FM, Ghaffar K, Chan YH, Amrun SN, Tan JJL, et al. Plasmodium co-infection protects against chikungunya virus-induced pathologies. Nat Commun. 2018;9(1):3905. https://doi.org/10.1038/ s41467-018-06227-9.

58. Muyanja E, Ssemaganda A, Ngauv P, Cubas R, Perrin H, Srinivasan D, et al. Immune activation alters cellular and humoral responses to yellow fever $17 \mathrm{D}$ vaccine. J Clin Invest. 2014;124(7):3147-58. https://doi.org/10. 1172/JCI75429.
59. Campi-Azevedo AC, Costa-Pereira C, Antonelli LR, Fonseca CT, Teixeira-Carvalho A, Villela-Rezende G, et al. Booster dose after 10 years is recommended following 17DD-YF primary vaccination. Hum Vaccin Immunother. 2016;12(2):491-502. https://doi.org/ $10.1080 / 21645515.2015 .1082693$.

60. Gotuzzo E, Yactayo S, Cordova E. Efficacy and duration of immunity after yellow fever vaccination: systematic review on the need for a booster every 10 years. Am J Trop Med Hyg. 2013;89(3):434-44. https://doi.org/ 10.4269/ajtmh.13-0264.

61. Poland JD, Calisher CH, Monath TP, Downs WG, Murphy K. Persistence of neutralizing antibody 30-35 years after immunization with $17 \mathrm{D}$ yellow fever vaccine. Bull World Health Organ. 1981;59(6):5.

62. Wieten RW, Jonker EFF, van Leeuwen EMM, Remmerswaal EBM, ten Berge IJM, de Visser AW, et al. A single 17D yellow fever vaccination provides lifelong immunity; characterization of yellow-fever-specific neutralizing antibody and T-cell responses after vaccination. Plos One. 2016;11(3). https://doi.org/10. 1371/journal.pone.0149871.

63. The World Health Organization. Lifetime validity of one dose of yellow fever vaccine: amendment to annex 7 of IHR (2005). International Health regulations: The World Health Organization2014.

64. Kareko BW, Booty BL, Nix CD, Lyski ZL, Slifka MK, Amanna IJ, et al. Persistence of neutralizing antibody responses among yellow fever virus $17 \mathrm{D}$ vaccinees living in a nonendemic setting. J Infect Dis. 2019;221:2018-25. https://doi.org/10.1093/infdis/ jiz374.

65. Collaborative group for studies on yellow fever v. Duration of post-vaccination immunity against yellow fever in adults. Vaccine. 2014;32(39):4977-84. https:// doi.org/10.1016/j.vaccine.2014.07.021.

66. Ndeffo-Mbah ML, Pandey A. Global risk and elimination of yellow fever epidemics. J Infect Dis. 2019;221:2026-34. https://doi.org/10.1093/infdis/ jiz375.

67. Patel D, Simons H. Yellow fever vaccination: is one dose always enough? Travel Med Infect Dis. 2013;11(5):266-73. https://doi.org/10.1016/j.tmaid. 2013.08.007.

68. Vannice K, Wilder-Smith A, Hombach J. Fractionaldose yellow fever vaccination - advancing the evidence base. N Engl J Med. 2018;379(7):603-5. https://doi. org/10.1056/NEJMp1803433.

69. Campi-Azevedo AC, de Almeida EP, Coelho-Dos-Reis JG, Peruhype-Magalhaes V, Villela-Rezende G, Quaresma PF, et al. Subdoses of 17DD yellow fever vaccine elicit equivalent virological:immunological kinetics timeline. BMC Infect Dis. 2014;14(391).

70. Roukens AH, Vossen AC, Bredenbeek PJ, van Dissel JT, Visser LG. Intradermally administered yellow fever vaccine at reduced dose induces a protective immune response: a randomized controlled non-inferiority trial. PLoS One. 2008;3(4):e1993. https://doi.org/10. 1371/journal.pone.0001993. 
71. Casey RM, Harris JB, Ahuka-Mundeke S, Dixon MG, Kizito GM, Nsele PM, et al. Immunogenicity of fractional-dose vaccine during a yellow fever outbreak final report. N Engl J Med. 2019;381(5):444-54. https://doi.org/10.1056/NEJMoa1710430.

72. Martins RM, Maia Mde L, Farias RH, Camacho LA, Freire MS, Galler R, et al. 17DD yellow fever vaccine: a double blind, randomized clinical trial of immunogenicity and safety on a dose-response study. Hum Vaccin Immunother. 2013;9(4):879-88. https://doi. org/10.4161/hv.22982.

73. Roukens AHE, van Halem K, de Visser AW, Visser LG. Long-term protection after fractional-dose yellow fever vaccination: follow-up study of a randomized, controlled. Noninferiority Trial Ann Intern Med. 2018;169(11):761-5. https://doi.org/10.7326/M181529.

74. de Menezes MR, Maia MLS, de Lima SMB, de Noronha TG, Xavier JR, Camacho LAB, et al. Duration of postvaccination immunity to yellow fever in volunteers eight years after a dose-response study. Vaccine. 2018;36(28):4112-7. https://doi.org/10.1016/j. vaccine.2018.05.041.

75. Gaucher D, Therrien R, Kettaf N, Angermann BR, Boucher G, Filali-Mouhim A, et al. Yellow fever vaccine induces integrated multilineage and polyfunctional immune responses. J Exp Med. 2008;205(13):311931. https://doi.org/10.1084/jem.20082292.

76. James EA, LaFond RE, Gates TJ, Mai DT, Malhotra U, Kwok WW. Yellow fever vaccination elicits broad functional $\mathrm{CD} 4+\mathrm{T}$ cell responses that recognize structural and nonstructural proteins. J Virol.

2013;87(23):12794-804. https://doi.org/10.1128/jvi. 01160-13.

77. Miller JD, van der Most RG, Akondy RS, Glidewell JT, Albott S, Masopust D, et al. Human effector and memory CD8+ T cell responses to smallpox and yellow fever vaccines. Immunity. 2008;28(5):710-22. https:// doi.org/10.1016/j.immuni.2008.02.020.

78. Veit O, Niedrig M, Chapuis-Taillard C, Cavassini M, Mossdorf E, Schmid P, et al. Immunogenicity and safety of yellow fever vaccination for $102 \mathrm{HIV}$-infected patients. Clin Infect Dis. 2009;48(5):659-66. https:// doi.org/10.1086/597006.

79. Monath TP, McCarthy K, Bedford P, Johnson CT, Nichols R, Yoksan S, et al. Clinical proof of principle for ChimeriVaxTM- recombinant live, attenuated vaccines against flavivirus infections. Vaccine. 2002;20:14.

80. Monath TP. Review of the risks and benefits of yellow fever vaccination including some new analyses. Expert Rev Vaccines. 2012;11(4):427-48. https://doi.org/10. 1586/erv.12.6.

81. Martin M, Weld L, Tsai TF, Mootrey GT, Chen RT, Niu $\mathrm{M}$, et al. Advanced age a risk factor for illness temporally associated with yellow fever vaccination. Emerg Infect Dis. 2001;7(6):7.

82. Khromava AY, Eidex RB, Weld LH, Kohl KS, Bradshaw RD, Chen RT, et al. Yellow fever vaccine: an updated assessment of advanced age as a risk factor for serious adverse events. Vaccine. 2005;23(25):3256-63. https://doi.org/10.1016/j.vaccine.2005.01.089.

83. Galler R, Pugachev KV, Santos CL, Ocran SW, Jabor AV, Rodrigues SG, et al. Phenotypic and molecular analyses of yellow fever 17DD vaccine viruses associated with serious adverse events in Brazil. Virology.

2001;290(2):309-19. https://doi.org/10.1006/viro. 2001.1168.

84. Vasconcelos PFC, Luna EJ, Galler R, Silva LJ, Coimbra TL, Barros VLRS, et al. Serious adverse events associated with yellow fever 17DD vaccine in Brazil: a report of two cases. Lancet. 2001;358(9276):91-7. https://doi. org/10.1016/s0140-6736(01)05326-0.

85. Suzano CE, Amaral E, Sato HK, Papaiordanou PM. Campinas group on yellow fever immunization during $P$. The effects of yellow fever immunization (17DD) inadvertently used in early pregnancy during a mass campaign in Brazil. Vaccine. 2006;24(9):1421-6. https://doi.org/10.1016/j.vaccine.2005.09.033.

86. Nasidi A, Monath TP, Vandenberg J, Tomori O, Calisher CH, Hurtgen X, et al. Yellow fever vaccination and pregnancy- a four-year prospective study. Trans $\mathrm{R}$ Soc Trop Med Hyg. 1993;87:2.

87. Bassi MR, Larsen MA, Kongsgaard M, Rasmussen M, Buus S, Stryhn A, et al. Vaccination with replication deficient adenovectors encoding YF-17D antigens induces long-lasting protection from severe yellow fever virus infection in mice. PLoS Negl Trop Dis. 2016;10(2):e0004464. https://doi.org/10.1371/ journal.pntd.0004464.

88. Maciel M Jr, Cruz Fda S, Cordeiro MT, da Motta MA, Cassemiro KM, Maia Rde C, et al. A DNA vaccine against yellow fever virus: development and evaluation. PLoS Negl Trop Dis. 2015;9(4):e0003693. https://doi.org/10.1371/journal.pntd.0003693.

89. Julander JG, Testori M, Cheminay C, Volkmann A. Immunogenicity and protection after vaccination with a modified Vaccinia virus Ankara-vectored yellow fever vaccine in the Hamster model. Front Immunol. 2018;9:1756. https://doi.org/10.3389/fimmu.2018. 01756.

90. Tottey S, Shoji Y, Jones RM, Chichester JA, Green BJ, Musiychuk K, et al. Plant-produced subunit vaccine candidates against yellow fever induce virus neutralizing antibodies and confer protection against viral challenge in animal models. Am J Trop Med Hyg. 2018;98(2):420-31. https://doi.org/10.4269/ajtmh. 16-0293.

91. Monath TP, Lee CK, Julander JG, Brown A, Beasley DW, Watts DM, et al. Inactivated yellow fever $17 \mathrm{D}$ vaccine: development and nonclinical safety, immunogenicity and protective activity. Vaccine. 2010;28(22):3827-40. https://doi.org/10.1016/j.vaccine.2010.03.023.

92. Kallas EG, D'Elia Zanella L, Moreira CHV, Buccheri R, Diniz GBF, Castineiras ACP, et al. Predictors of mortality in patients with yellow fever: an observational cohort study. Lancet Infect Dis. 2019;19(7):750-8. https://doi.org/10.1016/ S1473-3099(19)30125-2. 
93. Ho YL, Joelsons D, Leite GFC, Malbouisson LMS, Song ATW, Perondi B, et al. Severe yellow fever in Brazil: clinical characteristics and management. J Travel Med. 2019;26(5). https://doi.org/10.1093/jtm/taz040.

94. Mercorelli B, Palu G, Loregian A. Drug repurposing for viral infectious diseases: how far are we? Trends Microbiol. 2018;26(10):865-76. https://doi.org/10. 1016/j.tim.2018.04.004

95. Sacramento CQ, de Melo GR, de Freitas CS, Rocha N, Hoelz LV, Miranda M, et al. The clinically approved antiviral drug sofosbuvir inhibits Zika virus replication. Sci Rep. 2017;7:40920. https://doi.org/10.1038/ srep40920.

96. Xu HT, Colby-Germinario SP, Hassounah SA, Fogarty C, Osman N, Palanisamy N, et al. Evaluation of Sofosbuvir (beta-D-2'-deoxy-2'-alpha-fluoro-2' -betaC-methyluridine) as an inhibitor of dengue virus replication<sup/>. Sci Rep. 2017;7(1):6345. https:// doi.org/10.1038/s41598-017-06612-2.

97. Julander JG, Bantia S, Taubenheim BR, Minning DM, Kotian P, Morrey JD, et al. BCX4430, a novel nucleoside analog, effectively treats yellow fever in a Hamster model. Antimicrob Agents Chemother.

2014;58(11):6607-14. https://doi.org/10.1128/AAC. 03368-14.
98. de Freitas CS, Higa LM, Sacramento CQ, Ferreira AC, Reis PA, Delvecchio R, et al. Yellow fever virus is susceptible to sofosbuvir both in vitro and in vivo. PLoS Negl Trop Dis. 2019;13(1):e0007072. https://doi.org/ 10.1371/journal.pntd.0007072.

99. Mendes EA, Pilger DRB, Santos Nastri ACS, Malta FM, Pascoalino BDS, Carneiro D'Albuquerque LA, et al. Sofosbuvir inhibits yellow fever virus in vitro and in patients with acute liver failure. Ann Hepatol. 2019;18(6):816-24. https://doi.org/10.1016/j.aohep. 2019.09.001.

100. Lu X, Xiao H, Li S, Pang X, Song J, Liu S, et al. Double lock of a human neutralizing and protective monoclonal antibody targeting the yellow fever virus envelope. Cell Rep. 2019;26(2):438-46 e5. https://doi.org/10. 1016/j.celrep.2018.12.065.

\section{Publisher's Note}

Springer Nature remains neutral with regard to jurisdictional claims in published maps and institutional affiliations. 